\title{
Energy Distributions from Three-Body Decaying Many-Body Resonances
}

\author{
R. Álvarez-Rodríguez, A. S. Jensen, D. V. Fedorov, and H. O. U. Fynbo \\ Department of Physics and Astronomy, University of Aarhus, DK-8000 Aarhus C, Denmark \\ E. Garrido \\ Instituto de Estructura de la Materia, CSIC, Serrano 123, E-28006 Madrid, Spain
}

(Received 25 April 2007; published 17 August 2007)

\begin{abstract}
We compute energy distributions of three particles emerging from decaying many-body resonances. We reproduce the measured energy distributions from decays of two archetypal states chosen as the lowest $0^{+}$ and $1^{+}$resonances in ${ }^{12} \mathrm{C}$ populated in $\beta$ decays. These states are dominated by sequential, through the ${ }^{8} \mathrm{Be}$ ground state, and direct decays, respectively. These decay mechanisms are reflected in the "dynamic" evolution from small, cluster or shell-model states, to large distances, where the coordinate or momentum space continuum wave functions are accurately computed.
\end{abstract}

Introduction.-Energy and momentum conservation guarantees that two particles, emerging from decay of a given quantum state, appear with definite kinetic energies inversely proportional to their masses. In three-body decays the available energy can be continuously distributed among the particles. Prominent classical examples are $\alpha$ emission and $\beta$ decay, respectively. Surprisingly enough, the decay of a many-body quantum system into three particles has not been well described microscopically although discussed phenomenologically for various systems. The process depends on the initial state and the dynamic evolution, i.e., the decay mechanism.

This problem of three-body decay is common to several subfields of physics. The invention of Dalitz plots was an early attempt to classify the decay mechanisms by use of intermediate two-body doorway states [1]. The underlying dynamics in particle physics may be described as quark rearrangements. Similar decays occur in annihilation of a proton-antiproton pair from a Coulomb-like orbit into three mesons [2]. In molecular physics an example is decay of excited states of the $\mathrm{H}_{3}$ molecule into three hydrogen atoms [3]. In nuclear physics there exists a large number of three-body decaying systems of disparate structures and decay mechanisms, e.g., various excited states of ${ }^{6} \mathrm{He},{ }^{6} \mathrm{Li}$, ${ }^{12} \mathrm{C},{ }^{17} \mathrm{Ne}$. More and more high-quality experimental data have become available in all subfields [4-7], and quantitatively accurate models are needed to extract and understand the underlying physics.

The purpose of the present Letter is to compute the energy distributions for three-body decaying excited nuclear many-body resonances. We shall assume that the resonances are populated in $\beta$ decays and consequently only an outgoing flux is present. For reactions an ingoing flux is required to provide the population of the decaying wave function. Such a generalization is easily achieved by allowing initial conditions different from those of a resonance wave function. In all cases the major difficulty is to compute accurately the asymptotic large-distance three- body wave functions corresponding to genuine manybody resonances, which possibly differ at small distances from cluster states formed by the emerging three particles [8].

At least four problems must be solved: (i) the complex scaled resonance wave functions must be accurately determined even though they vanish exponentially at large distances, (ii) the wave functions must be traced as they "evolve dynamically" from relatively small to asymptotically large distances, (iii) the Coulomb problem of coupling continuum states at infinitely large distances must be solved, and (iv) the mixture of two- and three-body asymptotics must be accurately determined.

Theoretical framework.-We use the hyperspherical adiabatic expansion method of the Faddeev equations combined with complex scaling $[9,10]$. The hyperradius $\rho$ is the most important of the coordinates. For three identical particles of mass $m_{\alpha}$ the definition is

$$
m_{N} \rho^{2}=\frac{m_{\alpha}}{3} \sum_{i<j}^{3}\left(\boldsymbol{r}_{i}-\boldsymbol{r}_{j}\right)^{2}=m_{\alpha} \sum_{i=1}^{3}\left(\boldsymbol{r}_{i}-\boldsymbol{R}\right)^{2},
$$

where $\boldsymbol{r}_{i}$ is the coordinate of particle number $i, \boldsymbol{R}$ is the three-body center-of-mass coordinate, and $m_{N}=m_{\alpha} / 4$.

The two-body interactions are chosen to reproduce the available low-energy scattering data. A three-body potential with a range corresponding roughly to the three touching constituent particles is adjusted to reproduce the energy of each many-body resonance. The wave function and the complex energy of the resonance are then defined. Coordinate and momentum space angular wave functions are identical for a given total energy and an asymptotically large value of $\rho$ [11]. To obtain the energy $\left(E_{\alpha}\right)$ distribution of the particle we integrate the square of the resonance wave function over unobserved momenta.

The numerical computations must first provide accurate wave functions from small to intermediate distances, where the relatively fast changes due to the crucial short- 
range interaction are completed. This is efficiently achieved with the Faddeev decomposition, and a hyperspherical harmonics basis size individually adjusted to the accuracy needed for the different partial waves. The smoother variation from intermediate to asymptotic distances is analytical for short-range interactions [9]. The long-range Coulomb potentials can be treated numerically precisely as any other potential in the transitions from small to intermediate distances.

However, special treatment is required in the variations from intermediate to large distances. The necessary basis sizes become insurmountably large. Our solution is to compute an accurate wave function at intermediate, but relatively large, distances. This is achieved when an even larger hyperradius, compensated by a larger basis, leads to the same observables derived from the wave functions. This stability condition is difficult to reach when both three-body background continuum states are populated simultaneously with resonances in one or more of the two-body subsystems. At sufficiently large distances we can precisely identify these structures as components in the complex scaled wave functions related to different adiabatic potentials [11]; e.g., sequential decay proceeds through a potential approaching the corresponding complex two-body energy $E_{2 b}$ [10], whereas no intermediate structure is present for direct decay to the continuum.

When the two-body intermediate states have large widths the related radial wave function decreases quickly, because then the adiabatic couplings are large. These states then dissipate fast into the continuum described as direct decay and the distinction becomes artificial. This process eventually happens for all sequential decays since the intermediate states are unstable. Classification into sequential and direct decay is related to the use of different complete basis states, i.e., either two-body resonances and the third particle in the continuum or three-body continuum states. Interpretation as sequential or direct is then meaningful when a few states in one basis are sufficient while many are needed in the other. Such a distinction between paths producing the same observable is not possible in quantum mechanics.

Illuminating archetypes. -Accurate measurements of $\alpha$-particle energy distributions are available from decays of $0^{+}$and $1^{+}$resonances of ${ }^{12} \mathrm{C}[6,7,12,13]$. The lowest $0^{+}$ resonance is often described as a cluster state, whereas the $1^{+}$resonance in contrast is referred to as a shell-model state without any significant cluster structure [14-18]. Furthermore, the decay mechanisms are known to be different [6,7]. These cases are therefore ideally suited as illustrations of the present novel technique.

In Fig. 1 we show the lowest potentials where the attractive pockets at small distance support the resonances and provide the small-distance boundary conditions. As the hyperradius increases beyond the barriers, the potentials all decrease as $1 / \rho$ due to the Coulomb repulsion. The structure at large distances is necessarily of three-body character since this is the boundary condition imposed by the

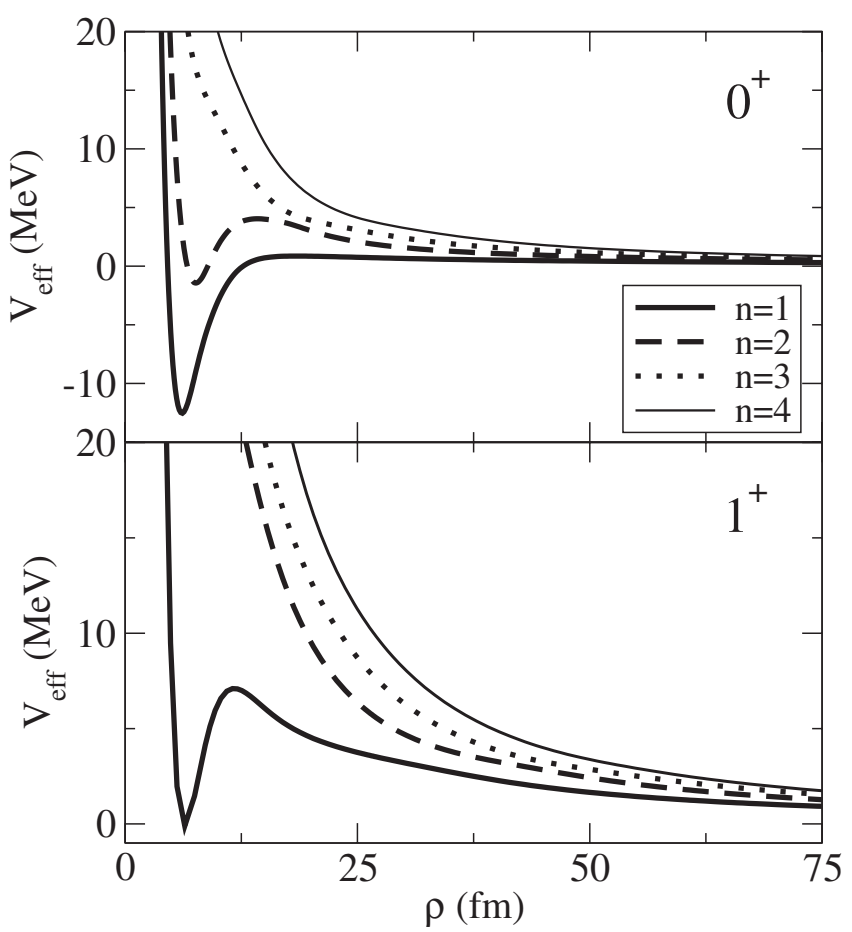

FIG. 1. The real parts of the four lowest adiabatic effective potentials, including the three-body potentials, as functions of $\rho$ for the $0^{+}$and $1^{+}$resonances of ${ }^{12} \mathrm{C}$. The two-body interaction, obtained from [19], is a slightly modified version of the a1 potential of [20]. The parameters of the three-body Gaussian potentials, $S \exp \left(-\rho^{2} / b^{2}\right)$, are $b=6 \mathrm{fm}$ and $-S=20,92 \mathrm{MeV}$ for $0^{+}$and $1^{+}$, respectively [21].

measurement. In contrast, at small distances these clusters overlap and the detailed description must use the nucleon degrees of freedom. The first adiabatic potential corresponds to the ${ }^{8} \mathrm{Be}\left(0^{+}\right)$state and therefore associated with this sequential decay. We shall explore the, perhaps surprising, conjecture that the decay can be described almost entirely within the present cluster model.

$1^{+}$resonance. - With the potentials in Fig. 1 we show the energy distribution in Fig. 2 for the $1^{+}$resonance where sequential decay via the ${ }^{8} \mathrm{Be}$ ground state is forbidden. The asymptotic behavior is reached for hyperradii larger than about $60 \mathrm{fm}$. The small variation of the distribution from 70 to $100 \mathrm{fm}$ shows the convergence and the stability. Higher accuracy is obtained at these distances with a moderate basis size than at larger distances where the basis quickly becomes insufficient. Two interfering adiabatic potentials are necessary to reach the impressive agreement with the measured distributions. It is remarkable that the cluster model provides this accuracy in spite of the fact that the initial decaying state is a many-body resonance without any three-body structure.

To test the reliability we Fourier transformed the wave function in two ways: first numerically with coordinates from $\rho=0$ to $100 \mathrm{fm}$ and secondly by use of the analytic solution obtained from the parametrized adiabatic potentials which asymptotically are sums of $1 / \rho$ and $1 / \rho^{2}$ 


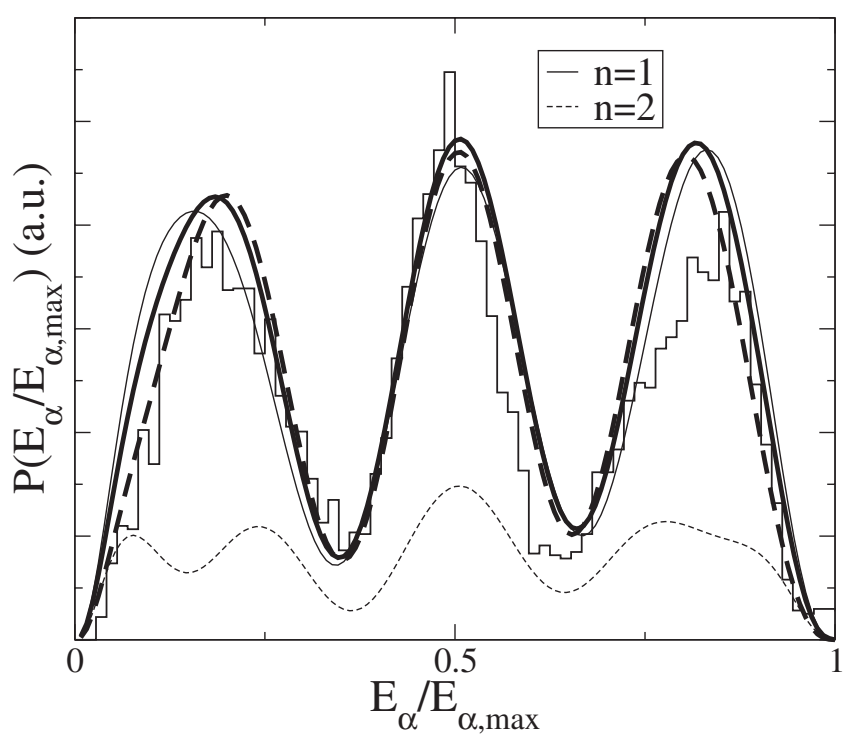

FIG. 2. The $\alpha$-particle energy distribution for the $1^{+}$resonance of ${ }^{12} \mathrm{C}$ at $5.43 \mathrm{MeV}$ above threshold at an excitation of $12.71 \mathrm{MeV}$. The energy is measured in units of the maximum possible, i.e., $2 \times 5.43 / 3 \mathrm{MeV}$. The thick solid curves and the dashed curves are for coordinate space wave functions at $\rho=$ $70,100 \mathrm{fm}$. The thin curves are contributions from separate adiabatic potentials. The histogram is the experimental distribution [13].

terms. The results are remarkably similar distributions and the analytic result is in fact indistinguishable from the curve for $\rho=100 \mathrm{fm}$ in Fig. 2. Small deviations from the measurements could be due to two-body interactions with resonance properties deviating slightly from the values measured for ${ }^{8} \mathrm{Be}$. However, most of the differences are more likely due to uncertainties arising from acceptance of the detectors used in the experiment.

It is amusing to estimate that the sequential decay via the ${ }^{8} \mathrm{Be} 2^{+}$resonance would produce a similar central peak of about twice the width. To reproduce the data strong interference would then be necessary. Good phenomenological reproduction of the data is obtained by $R$-matrix theory where the smaller width is explained due to preferentially populating the low-energy tail of the ${ }^{8} \mathrm{Be} 2^{+}$resonance, and where effects of interference also play an important role [6].

$0^{+}$resonances. - The complex scaled radial wave functions are shown in Fig. 3 for the two lowest $0^{+}$resonances. The largest probability is found at small distance and they all vanish with increasing hyperradius. Their relative sizes are fairly insensitive to variations of the hyperradius at large distances where the energy distributions are determined. The first resonance is described by the first adiabatic component for all distances whereas the second resonance changes character from small to large $\rho$ from $4 \%$ to $75 \%$ of the first adiabatic component. This component approaches the configuration of the $0^{+}$resonance in ${ }^{8} \mathrm{Be}$ with the third $\alpha$ particle far away, i.e., $s$ waves between each pair of $\alpha$ particles for each of the three Faddeev

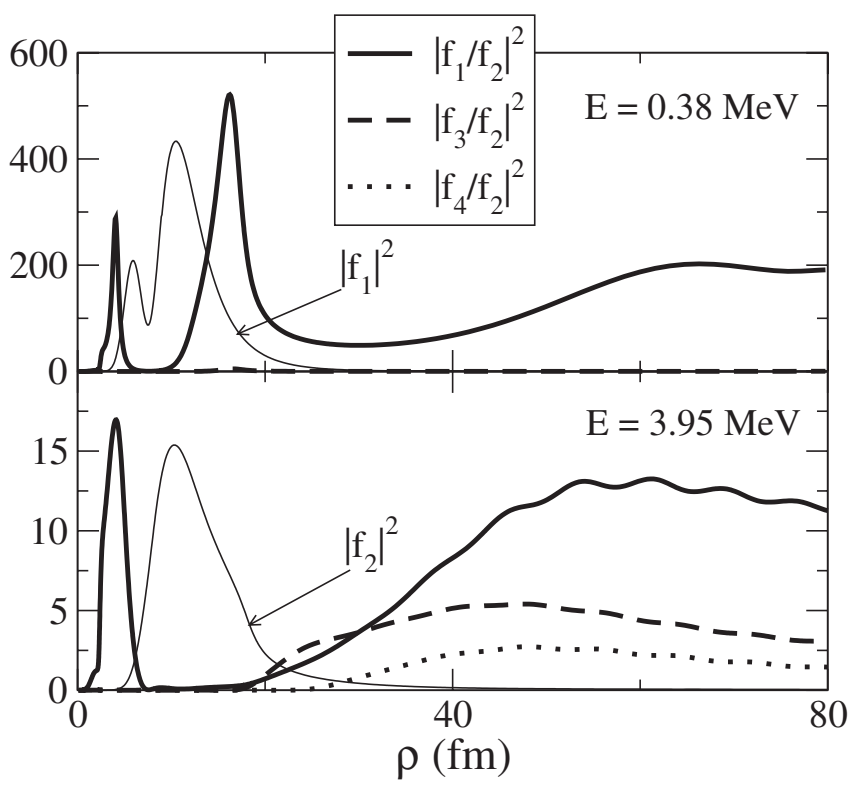

FIG. 3. Ratios and the small-distance dominating wave functions as functions of $\rho$ for each of the two $0^{+}$resonances of ${ }^{12} \mathrm{C}$ at 0.38 and $3.95 \mathrm{MeV}$ above threshold or at excitation energies of 7.63 and $11.2 \mathrm{MeV}$ [13].

components. The result is an energy distribution with characteristic features of sequential three- $\alpha$ decay: a narrow high-energy peak and a distribution around one quarter of the maximum of the ${ }^{12} \mathrm{C}$ resonance energy. Unfortunately, these computed distributions are not accurate because the two-body asymptotic behavior in these cases is not reached for $\rho$ less than $100 \mathrm{fm}$. However, the method provides the amount of sequential decay and we can substitute the inaccurate component by the known twobody asymptotic behavior. The energy distribution from decay of the first $0^{+}$resonance at $0.38 \mathrm{MeV}$ is then seen from Fig. 3 to be almost entirely determined by the first potential, which means sequential decay. The direct decay is about $1 \%$ in agreement with the experimental upper limit [12]. This energy distribution is then in complete agreement with experimental data.

The second $0^{+}$resonance is also dominated by the first adiabatic potential at large distance. This is in striking contrast to the domination by the second potential at small distance. This is an example of the importance of the dynamical evolution from small to large distances. The result is about $75 \%$ sequential (first potential) and 25\% direct decay described by the other adiabatic potentials. In comparison with measurements complications arise for two reasons, both related to the large width of the order $1 \mathrm{MeV}$. First, effects of energy-dependent feeding in the $\beta$ decay populating the decaying state are substantial in the data $[7,13]$. Higher $\beta$ energies are rather strongly favored resulting in distributions moving towards lower energies. Second, the experimental analysis is hampered by possible effects from other resonances. Their contributions are possibly not fully disentangled. 


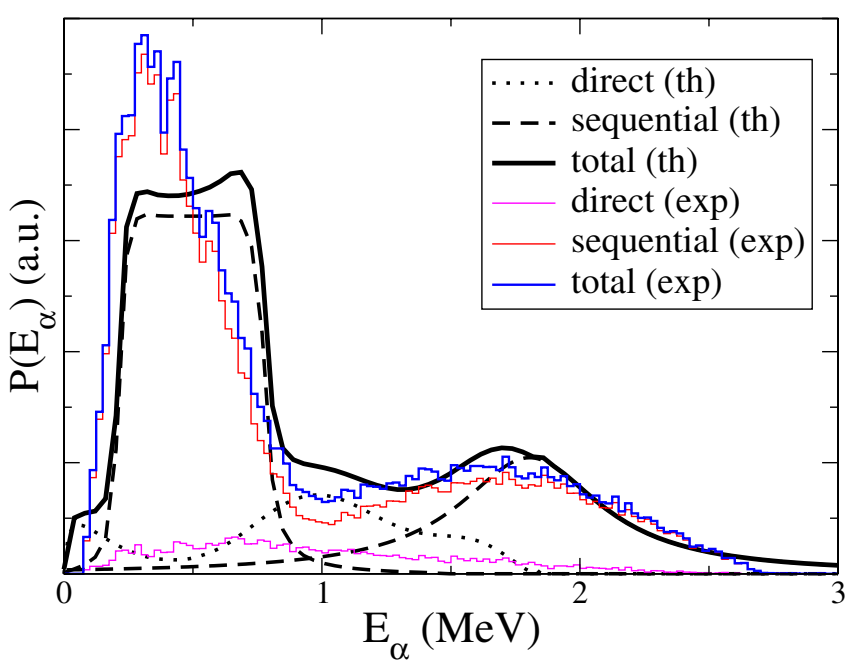

FIG. 4 (color online). The $\alpha$-particle energy distribution for the second $0^{+}$resonance of ${ }^{12} \mathrm{C}$ at $4.3 \mathrm{MeV}$ above threshold at an excitation of $11.2 \mathrm{MeV}[7,13]$. The interactions in Fig. 1 give an energy of $3.95 \mathrm{MeV}$ above threshold [21]. We use $2.8 \mathrm{MeV}$ to account for interference and $\beta$-feeding distortion. The maximum energy for the most likely resonance position is then $2 \times$ $2.8 / 3 \approx 1.8 \mathrm{MeV}$. The histograms are experimental direct (the small part), sequential, and total distribution.

The peak energy corresponding to the resonance position is at about $2.8 \mathrm{MeV}$ in the $\beta$-feeding process $[7,13]$. We illustrate in Fig. 4 the sequential part of the energy distribution of the first emitted $\alpha$ particle by using the Breit-Wigner distribution defined with the most probable position at $2.8 \mathrm{MeV}$ and a width equal to the sum of the widths of the three-body decaying resonance and the intermediate two-body resonance. The two $\alpha$ particles following from decay of ${ }^{8} \mathrm{Be}$ are uniquely related by kinematic conditions resulting in a peak at lower energy. The large width of the three-body decaying resonance smears out the latter distribution. Between these two peaks appears the contribution of about $25 \%$ from direct decay described by the other adiabatic potentials. The inaccuracies in the computed distributions are, first, that deviations from the Breit-Wigner shape become important for the large width of $1 \mathrm{MeV}$, and second that the fraction of sequential decay may be underestimated by perhaps $10 \%$ due to missing higher partial waves.

In any case, the shape of the sequential decay via the ${ }^{8} \mathrm{Be}$ ground state is derived by precisely the same kinematic conditions in both the computation and the experimental analysis. The largest differences between theory and experiment are simulated by the shift of resonance peak energy. The agreement is rather good and only possible due to the computed decay mechanism of dynamic evolution with hyperradius.

Summary and conclusions. - We have computed the energy distributions for three-body decaying many-body resonances. Combinations of short-range and repulsive Coulomb interactions are allowed. We conjecture, and show in specific cases, that the energy distributions of the decay fragments are insensitive to the short-distance manybody structure, but accessible in a three-body cluster model. The resonance structures may be completely different at small and large distances. This dynamic evolution is decisive for the decay mechanism. We separate components with two- and three-body asymptotics corresponding to sequential and direct decays. This distinction is crucial to obtain accurate wave functions at large distances. We test the method by comparing results from coordinate and momentum space.

We illustrate by application to the archetypes of $\alpha$-decaying $0^{+}$and $1^{+}$states in ${ }^{12} \mathrm{C}$. The $1^{+}$resonance cannot be described as a three-body state but its decay proceeds directly into the three-body continuum. The two $0^{+}$resonances both have substantial, but very different, cluster components at small distances. However, they both decay preferentially through the same large-distance structure best described as the $0^{+}$resonance of ${ }^{8} \mathrm{Be}$. These sequential decays imply a total rearrangement of the second of these resonances from small to large distances. The accurate $\alpha$-particle energy distributions for all three resonances populated in $\beta$ decay are reproduced remarkably well. Thus the method has passed very severe tests. It is reliable and with predictive powers.

[1] R. H. Dalitz, Philos. Mag. 44, 1068 (1953).

[2] C. Amsler, Rev. Mod. Phys. 70, 1293 (1998).

[3] U. Galster, U. Müller, and H. Helm, Phys. Rev. Lett. 92, 073002 (2004).

[4] U. Galster, F. Baumgartner, U. Müller, H. Helm, and M. Jungen, Phys. Rev. A 72, 062506 (2005).

[5] B. Blank et al., C.R. Physique 4, 521 (2003).

[6] H. O. U. Fynbo et al., Phys. Rev. Lett. 91, 082502 (2003).

[7] C. Aa. Diget et al., Nucl. Phys. A760, 3 (2005).

[8] E. Garrido, D. V. Fedorov, H. O. U. Fynbo, and A.S. Jensen, Nucl. Phys. A781, 387 (2007).

[9] E. Nielsen, D. V. Fedorov, A. S. Jensen, and E. Garrido, Phys. Rep. 347, 373 (2001).

[10] D. V. Fedorov, E. Garrido, and A.S. Jensen, Few-Body Syst. 33, 153 (2003).

[11] E. Garrido, D. V. Fedorov, A.S. Jensen, and H.O. U. Fynbo, Nucl. Phys. A766, 74 (2006).

[12] M. Freer et al., Phys. Rev. C 49, R1751 (1994).

[13] C. Aa. Diget, Ph.D. thesis, University of Aarhus, 2006.

[14] Y. Kanada-En'yo, Phys. Rev. Lett. 81, 5291 (1998).

[15] T. Neff and H. Feldmeier, Nucl. Phys. A738, 357 (2004).

[16] P. Descouvemont, Nucl. Phys. A709, 275 (2002).

[17] S. C. Pieper, Nucl. Phys. A751, 516 (2005).

[18] P. Navratil, J. P. Vary, and B. R. Barrett, Phys. Rev. C 62, 054311 (2000).

[19] D. V. Fedorov and A.S. Jensen, Phys. Lett. B 389, 631 (1996).

[20] S. Ali and A. R. Bodmer, Nucl. Phys. 80, 99 (1966).

[21] R. Álvarez-Rodríguez, E. Garrido, A.S. Jensen, D. V. Fedorov, and H.O. U. Fynbo, Eur. Phys. J. A 31, 303 (2007). 